\title{
Acamprosate-induced Extrapyramidal Symptoms in an Elderly Patient with Alcohol Dependence
}

\author{
Jungmin Woo', Hyo-Deog Rim $^{2}$ \\ 'Department of Psychiatry, Catholic University of Daegu School of Medicine, ${ }^{2}$ Department of Psychiatry, Kyungpook National University \\ School of Medicine, Daegu, Korea
}

\begin{abstract}
Acamprosate reduces the craving for alcohol by decreasing glutamate activity and increasing gamma-aminobutyric acid (GABA) action in patients with alcohol dependence. Acamprosate has tolerable side effects that include diarrhea, headache, dizziness and pruritus. In this study, we report acamprosate-induced extrapyramidal symptoms in an elderly patient with no history of neurologic disease. Severe extrapyramidal symptoms developed two days after the administration of acamprosate and improved over one week after the acamprosate was stopped. Extrapyramidal symptoms are commonly associated with dopamine receptor antagonists. However, there have been several reports of extrapyramidal symptoms occurring with drugs targeting other systems, including GABA, glutamate and serotonin. Acamprosate may decrease dopamine levels in the ventral tegmental area mediated by glutamatergic action and thus cause extrapyramidal symptoms. We suggest that acamprosate carries the risk of causing extrapyramidal symptoms.
\end{abstract}

KEY WORDS: Acamprosate; Extrapyramidal symptoms; Glutamate; Gamma-aminobutyric acid.

\section{INTRODUCTION}

Acamprosate (calcium acetylhomotaurinate) is a derivative of the amino acid taurine, and its structure is similar to that of gamma-aminobutyric acid (GABA). Acamprosate interacts with the glutamate and GABA systems. In patients with chronic alcohol exposure, glutamate hyperactivity and GABA deficiency explain some of the mechanisms of craving; acamprosate reduces these cravings by decreasing the glutamate activity and increasing the action of GABA. ${ }^{1)}$

Acamprosate has been proven safe and effective for the treatment of alcoholism and is approved by US Food and Drug Administration. Some of the common side effects of acamprosate are diarrhea, headache, dizziness and pruritus. ${ }^{2)}$ Although most cases of drug-induced extrapyramidal symptoms (EPSs) are associated with antipsychotics targeting the dopamine system, EPSs can also develop due to drugs targeting other systems, including selective

\footnotetext{
Received: June 12, 2014 / Revised: July 18, 2014

Accepted: July 22, 2014

Address for correspondence: Hyo-Deog Rim, MD, PhD

Department of Psychiatry, Kyungpook National University School of

Medicine, 130 Dongdeok-ro, Jung-gu, Daegu 700-721, Korea

Tel: +82-53-420-5755, Fax: +82-53-426-5361

E-mail: hdrim@knu.ac.kr
}

serotonin reuptake inhibitors. ${ }^{3)}$ The following is a case of acamprosate-induced EPSs in an elderly patient with no history of neurologic disease.

\section{CASE}

A 72-year-old unemployed, married man entered treatment to stop alcohol intake. He had consumed alcohol regularly for ten years. His history revealed no prior hospitalizations and no experience of severe withdrawal symptoms. The patient demonstrated a mild tremor of both hands, a pulse of 79 beats per minute, a respiratory rate of 20 per minute, and blood pressure of 110/70. All blood tests were within normal limits.

He was diagnosed with alcohol dependency. He was detoxified with lorazepam $4 \mathrm{mg} /$ day for 4 days and was prescribed $999 \mathrm{mg}$ /day of acamprosate and $100 \mathrm{mg} /$ day of thiamine. After 2 days, he exhibited a slowed gait with diminished pendular arm movements, excessive saliva pooling in his mouth, muscle rigidity of arms without cogwheel rigidity and bradykinesia with slowed speech. However, there were no focal signs in a neurological examination. Over the next 2 days, acute onset Parkinsonism developed and prohibited him from daily activity. Acamprosate was discontinued, and the symp-

(a) This is an Open-Access article distributed under the terms of the Creative Commons Attribution Non-Commercial License (http://creativecommons.org/licenses/by-nc/3.0) which permits unrestricted non-commercial use, distribution, and reproduction in any medium, provided the original work is properly cited. 


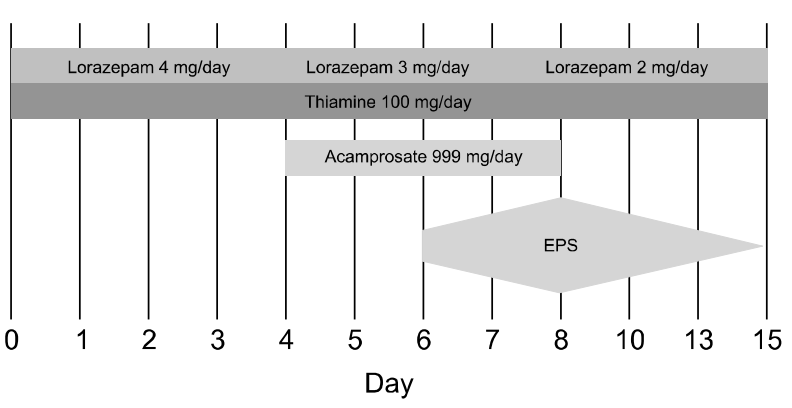

Fig. 1. Administered medications and the course of extrapyramidal symptoms. EPSs, extrapyramidal symptoms.

toms disappeared over the next week (Fig. 1).

\section{DISCUSSION}

This is the second case report of a patient with alcohol dependence who developed EPSs following the administration of acamprosate. This patient experienced severe EPSs two days after administration of acamprosate, which continued for nine days. We did not initially recognize these unexpected EPSs as side effects; we explored the possibility of acute-onset neurological disease and researched drugs that may have been responsible for the EPSs. However, there were no signs of neurological disease, and we found no drugs that had EPSs as common side effect. Acamprosate was discontinued, and the EPSs disappeared over the following week.

Previously, acamprosate-induced EPSs were reported in a 36-year-old patient with alcohol dependence. ${ }^{4)}$ The patient took 1,998 mg/day of acamprosate for one week before the EPSs developed. The dose was reduced to $1,332 \mathrm{mg} /$ day and continued at this level for one week. Nevertheless, the symptoms remained. The EPSs did not end until the medication was discontinued.

It is important to compare the currently reported case with the previous case from a clinical perspective, despite a lack of information. We prescribed reduced dose of acamprosate ( $999 \mathrm{mg} /$ day) because the patient was elderly (72 years) with a low body weight $(51 \mathrm{~kg})$. The EPSs developed over two days, while in the previous case, the dose was 1,998 $\mathrm{mg}$ and the EPS developed after one week. However, symptoms disappeared in both cases after acamprosate was discontinued. Thus, acamprosate-induced EPSs developed at half the recommended dose and more quickly in the elderly patient. The sensitivity to this side effect may be due advanced the patient's age and altered pharmacokinetics. Acamprosate is eliminated via the kidney and must be prescribed at a reduced dose or not at all in patients with renal impairment, depending on the severity of the impairment. Although we could not find any proof, our elderly patient may have had decreased renal function and therefore sensitivity to this side effect. In both cases, it took approximately one week for the EPSs to disappear after the drug was discontinued. In the previous case, dose reduction only attenuated the symptoms. Discontinuing the medication may be the better strategy when EPSs occur, and the disappearance of symptoms may take a considerable length of time, especially in elderly patients. The mean maximum plasma concentration $\left(\mathrm{C}_{\max }\right)$ of acamprosate was $180 \mathrm{ng} / \mathrm{ml}$ after oral administration of a single dose of $666 \mathrm{mg}$ in healthy subjects. ${ }^{5)}$ Acamprosate reaches steady-state plasma concentrations after 5 to 7 days of treatment and has an elimination half-life of approximately 30 hours. ${ }^{6}$ In the case of an individual with a possible decrement in renal functioning, such as an elderly patient, the side effects can last longer because $\mathrm{C}_{\max }$ can be higher, time to $\mathrm{C}_{\max }$ can be longer and plasma elimination half-life can be significantly longer than in a healthy subject.

The pathophysiology of drug-induced EPSs involves decreased dopamine activity in the basal ganglia. Therefore, dopamine receptor antagonists are commonly associated with EPSs. Their affinity to $\mathrm{D}_{2}$ receptors in the basal ganglia is particularly associated with such symptoms. Acamprosate affects the GABA and glutamate system. Acamprosate-induced EPSs are very rare, and the mechanism underlying their appearance is unknown. However, a clue may lie in the glutamate system. The site of acamprosate action, the ventral tegmental area, is highly complex and heterogeneous: it contains dopaminergic, GABAergic and glutamatergic neurons. It has been suggested that the local glutamatergic neurons regulate the neuronal network of the ventral tegmental area. ${ }^{7)}$ Most dopamine neurons in this area show glutamate reactivity, and dopamine release is affected by this action. ${ }^{8)}$ The excitatory synaptic responses of dopamine neurons are mediated by glutamate, which leads to dopamine release and the inhibition of excitatory responses via presynaptic $\mathrm{D}_{2}$ receptors. Thus, acamprosate may diminish glutamate activity in this area, which decreases dopamine and leads to EPSs. Alcohol withdrawal can induce decrements in dopamine release in the ventral tegmental area, ${ }^{9)}$ and there may be additive effects with acamprosate administration that affect the development of extra pyramidal symptoms.

This is the second case report of the development of EPSs with acamprosate administration. It may be controversial to make conclusions based on two cases, espe- 
cially when the elderly patient described in this report might have had a transient condition evoking EPSs. Nonetheless, this report suggests that acamprosate carries the risk of causing EPSs, and clinicians should be aware of this possibility. Future studies should be conducted using a placebo-controlled prospective design with precise evaluations during initial two weeks of acamprosate treatment.

\section{REFERENCES}

1. Hunt WA. Neuroscience research: how has it contributed to our understanding of alcohol abuse and alcoholism? A review. Alcohol Clin Exp Res 1993;17:1055-1065.

2. Bouza C, Angeles M, Muñoz A, Amate JM. Efficacy and safety of naltrexone and acamprosate in the treatment of alcohol dependence: a systematic review. Addiction 2004;99: 811-828.

3. Madhusoodanan S, Alexeenko L, Sanders R, Brenner R. Extrapyramidal symptoms associated with antidepressants--a review of the literature and an analysis of spontaneous reports. Ann Clin Psychiatry 2010;22:148-156.

4. Sidana AK, Mangla D. Unusual side effects with acamprosate. Indian J Psychiatry 2007;49:143.

5. Scott LJ, Figgitt DP, Keam SJ, Waugh J. Acamprosate: $a$ review of its use in the maintenance of abstinence in patients with alcohol dependence. CNS Drugs 2005;19:445-464.

6. Saivin S, Hulot T, Chabac S, Potgieter A, Durbin P, Houin G. Clinical pharmacokinetics of acamprosate. Clin Pharmacokinet 1998;35:331-345.

7. Morales M, Root DH. Glutamate neurons within the midbrain dopamine regions. Neuroscience 2014. doi: 10.1016/j.neuroscience. 2014.05.032. [Epub ahead of print].

8. Rayport S. Glutamate is a cotransmitter in ventral midbrain dopamine neurons. Parkinsonism Relat Disord 2001;7:261264.

9. Diana M, Pistis M, Carboni S, Gessa GL, Rossetti ZL. Profound decrement of mesolimbic dopaminergic neuronal activity during ethanol withdrawal syndrome in rats: electrophysiological and biochemical evidence. Proc Natl Acad Sci U S A 1993;90:7966-7969. 